\title{
Incorporación del urianálisis como técnica laboratorial en el abordaje diagnóstico de casos clinicos (ABP) para la adquisición de competencias profesionales
}

\author{
Laura Navarro ${ }^{a}$, Maria Teresa Verde ${ }^{b}$, Cristina Arbizu \\ aprofesora Asociada. Departamento de Patología Animal. Facultad de Veterinaria. Universidad de \\ Zaragoza. launav@unizar.es, bProfesora Titular. Departamento de Patología Animal. Facultad de \\ Veterinaria. Universidad de Zaragoza. mverde@unizar.es, 'Técnico de Laboratorio. Hospital \\ Veterinario. Universidad de Zaragoza. crisalbi@unizar.es
}

\begin{abstract}
Problem-based learning (PBL) is one of the most used methodologies in the teaching of medical disciplines such as veterinary medicine, since it allows applying the knowledge acquired in a real context. In addition, urinalysis is a laboratory technique essential in the veterinary clinical practice for the diagnostic approach of clinical cases. Therefore, we set out to carry out an experience with two objectives: 1) to promote the acquisition of skills in the laboratory, especially in urinalysis, and 2) to promote the acquisition of competencies by integrating the results of urinalysis in the diagnostic approach of a real case. The experience was carried out with students of the last courses of the veterinary degree at the Veterinary Hospital of Zaragoza. The students assessed various aspects of the proposed objectives through a survey. 47 students participated, valuing the experience as very satisfactory. The majority perceived to have increased their skills in the laboratory by performing the urinalysis technique, and considered as very useful the integration of urinalysis results in the diagnostic approach of the cases, considering in general, that the knowledge acquired could be very useful in their professional future.
\end{abstract}

Keywords: problem-based learning, last courses, skills, veterinary medicine, clinical cases, urinalysis, laboratory.

\section{Resumen}

El aprendizaje basado en problemas (ABP) es una de las metodologías más empleadas en la docencia de disciplinas biomédicas como la medicina interna veterinaria, ya que permite afianzar el aprendizaje de una forma muy sólida aplicando los conocimientos previos adquiridos en materias más básicas a un contexto real. Permite a los estudiantes poner en valor el conocimiento adquirido previamente y da sentido a los planteamientos académicos, que, de otra forma, no acaban de ser entendidos por los propios estudiantes. Por otro lado, el urianálisis es una técnica laboratorial imprescindible en la práctica clínica veterinaria para el abordaje diagnóstico de todos los casos clínicos relacionados con enfermedades del sistema urinario, pero que, además, 
coplementa la valoración de la situación global de cualquier proceso orgánico. Por ello, nos propusimos realizar una experiencia con dos objetivos: 1) fomentar la adquisición de habilidades en el laboratorio, especialmente en el urianálisis y 2) fomentar la adquisición de competencias integrando los resultados del urianálisis en el abordaje diagnóstico de un caso real. La experiencia se llevó a cabo con alumnos/as de últimos cursos del grado de Veterinaria en el Hospital Veterinario de la Universidad de Zaragoza. El alumnado evaluó, mediante una encuesta, distintos aspectos sobre los objetivos planteados. 47 alumnos/as participaron valorando la experiencia como muy satisfactoria. La mayoría percibió haber aumentado sus destrezas en el laboratorio mediante la realización de la técnica de urianálisis, y valoró como muy útil la integración de los resultados del urianálisis en el abordaje diagnóstico de los casos, considerando en general, que los conocimientos adquiridos podrían serles muy útiles en su futuro profesional y poniendo mucho mas en valor los conocimientos que poseían de la materia relacionada de cursos precedentes, lo que se traduce en un incremento de su autoestima al hacerse conscientes de la proyección real que pueden tener los conocimientos adquiridos.

Palabras clave: aprendizaje basado en problemas, últimos cursos, competencias, veterinaria, casos clínicos, urianálisis, laboratorio.

\section{Introducción}

El ABP o aprendizaje basado en problemas es uno de los sistemas educativos más extensamente investigados y experimentados, ya que fomenta el aprendizaje constructivista, donde el alumno aprende construyendo a partir de lo que sabe; y el aprendizaje cooperativo, promoviendo el trabajo en equipo, que es una de las maneras más eficaces para aprender (Barrows, 1980). La incorporación de este tipo de metodologías activas ha sido ampliamente utilizada en la docencia de disciplinas biomédicas como enfermería, medicina o veterinaria, debido a que promueven una formación "integral" del alumnado, permitiendo la adquisición de competencias específicas y transversales que le van a ser muy útiles en su futuro profesional (Walton, 1989). Por un lado, hacen que el alumno se enfrente a problemas clínicos reales similares a los que se encontrará cuando desarrolle su profesión. Por otro lado, fomenta la adquisición de competencias transversales como la capacidad de análisis y síntesis, el espíritu crítico y la toma de decisiones, al tener que aplicar los conocimientos teóricos adquiridos en la resolución de un caso práctico (Escanero, 2002).

Hace más de diez años que este tipo de metodologías activas se integran dentro de la docencia práctica y teórica de algunas de las asignaturas de ámbito clínico del Grado de Veterinaria en la Universidad de Zaragoza. El alumnado de los últimos cursos, se enfrenta a situaciones prácticas con casos reales o simulados, donde hace un ejercicio de análisis y aplicación de los conocimientos teóricos y prácticos que han ido adquiriendo a lo largo de sus estudios. Además, en ocasiones tienen que poner en práctica destrezas y habilidades que 
han tenido escasa oportunidad de desarrollar a lo largo de su formación, como pueden ser la extracción de sangre a un paciente en la consulta, o la realización una prueba laboratorial como el análisis de una muestra de orina.

En la práctica clínica veterinaria es muy importante adquirir experiencia en la realización e interpretación de los resultados de pruebas laboratoriales como el urianálisis, que permiten obtener información fundamental dentro del abordaje diagnóstico de los problemas clínicos que afronta el veterinario en la consulta. El urianálisis es especialmente importante en el abordaje de los casos clínicos relacionados con patologías del aparato urinario, aunque también ayuda a orientar situaciones clínicas en las que hay alteraciones de otros sistemas u órganos (Carolyn, 2012). A pesar de su utilidad, la técnica de urianálisis tiene poco protagonismo entre las pruebas laboratoriales que se realizan en el ámbito clínico. Por ello, resulta fundamental fomentar en el alumnado de los últimos cursos del grado de Veterinaria, el empleo de esta técnica, para que: por un lado, sean capaces de realizarla correctamente, utilizando los nuevos equipos laboratoriales automáticos actualmente disponibles en la práctica clínica; y por otro lado, puedan analizar e interpretar los resultados obtenidos dentro del abordaje diagnóstico de un caso clínico; adquiriendo así importantes competencias/habilidades que tendrán que desarrollar en su futuro profesional.

\section{Objetivos}

Ante la necesidad de formar a los alumnos/as de últimos cursos del grado de Veterinaria en la adquisición de competencias/habilidades del ámbito clínico dentro de una formación integral, y como profesorado que imparte una gran parte de su docencia en asignaturas de carácter clínico, propusimos el diseño de esta experiencia con los siguientes objetivos:

1. Fomentar el trabajo autónomo del alumno en la adquisición de destrezas y habilidades en el laboratorio, especialmente en la técnica de urianálisis, con el empleo del nuevo equipamiento actualmente disponible en el ámbito clínico.

2. Fomentar la adquisición de competencias transversales y profesionalizantes, mediante el abordaje diagnóstico de un caso-problema real, integrando, analizando e interpretando los resultados laboratoriales obtenidos.

\section{Desarrollo de la innovación}

Esta experiencia se llevó a cabo durante la docencia práctica de las asignaturas de Integración de Animales de Compañía (IACOM) de $4^{\circ}$ curso y Prácticum de Pequeños Animales, Exóticos y Equidos de $5^{\circ}$ curso, entre los meses de octubre y marzo del curso 2018-2019. Se desarrolló en las dependencias del Hospital Veterinario de la Universidad de Zaragoza (HVZ): en el laboratorio y en las consultas donde se atendieron a los pacientes que acudieron tanto al Servicio de Medicina Interna como al Servicio de Urología y Nefrología de este hospital. 
Las actividades que se realizaron se exponen a continuación en un orden cronológico:

\subsection{Trabajo en el laboratorio:}

\subsubsection{Realización de la técnica de urianálisis}

Los estudiantes realizaron la técnica de urianálisis trabajando en pequeños grupos (1-3 personas) y de forma autónoma, supervisados por el/la tutor/a. Para ello emplearon las muestras de orina obtenidas de los pacientes que fueron atendidos en las consultas del HVZ mediante técnicas manuales tradicionales y con el último equipamiento disponible en el laboratorio.

\subsubsection{Recogida de los resultados obtenidos}

Posteriormente, recogieron los resultados obtenidos en una hoja-informe prevista para este fin (Anexo I).

\subsection{Trabajo en la consulta:}

\subsubsection{Interpretación de los resultados y discusión}

En la consulta y de forma grupal y autónoma (sin el tutor), procedieron al análisis e interpretación de los resultados de la hoja informe del urianálisis extrayendo una serie de conclusiones sobre los mismos.

\subsubsection{Integración de los resultados en el abordaje diagnóstico del caso clínico}

Posteriormente y junto con el/la tutor/a, se pusieron en común las conclusiones sobre los resultados obtenidos en el urianálisis y se interpretaron dentro del abordaje diagnóstico del cuadro clínico que presentaba el paciente, analizando y discutiendo el posible orígen del mismo. Se determinaron los siguientes pasos a seguir dentro del abordaje del caso y/o las medidas terapéuticas a tomar en función de los resultados obtenidos. Todas las decisiones adoptadas se pusieron en conocimiento de los propietarios posteriormente.

\subsubsection{Evaluación de la experiencia}

Al final de la práctica, los alumnos y el profesorado, completaron una encuesta tipo google desde el ordenador de la consulta, en la que evaluaron según una escala de Likert de 5 puntos distintos aspectos relacionados con la sesión práctica, especialmente aquellos relacionados con la adquisición de competencias (Anexo II).

\section{Resultados}

Un total de 47 alumnos/as realizaron esta experiencia. Quince $(31,9 \%)$ alumnos/as de $4^{\circ}$ curso la hicieron durante las sesiones prácticas de la asignatura de "Integración de Animales de Compañía" que se desarrollaron en la consulta de medicina interna del HVZ y donde se atendieron a pacientes con distintas patologías. Treinta y dos $(68,1 \%)$ alumnos/as de $5^{\circ}$ curso la hicieron durante las sesiones prácticas de la asignatura "Practicum de Pequeños Animales, Exóticos y Equidos", en la consulta de la especialidad de Urología del $\mathrm{HVZ}$, donde se atendieron a pacientes con patologías relacionadas con el aparato urinario 
(Figura 1). Del total del alumnado, treinta y tres $(70,2 \%)$ fueron mujeres y catorce $(29,8 \%)$ fueron hombres (Figura 2).

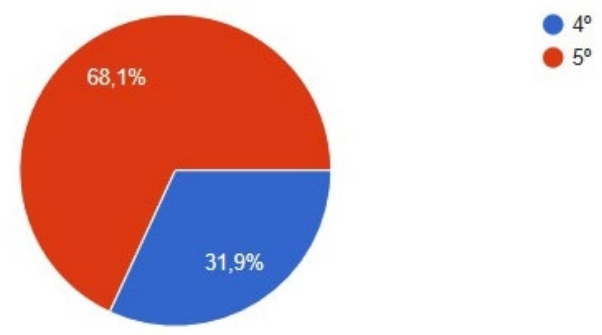

Figura 1. Distribución del alumnado en función del curso académico

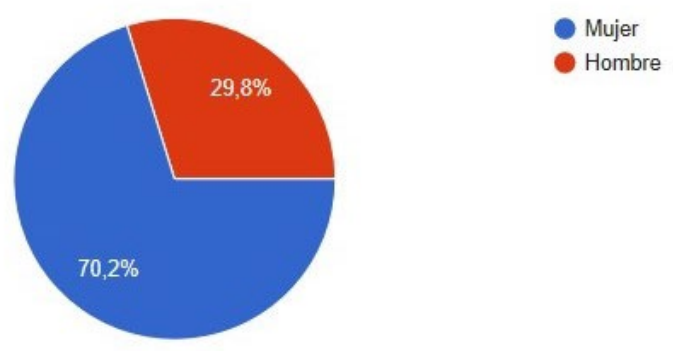

Figura 2. Distribución del alumnado en función del género

La Figura 3 representa la edad del alumnado en el momento en el que realizó la práctica.

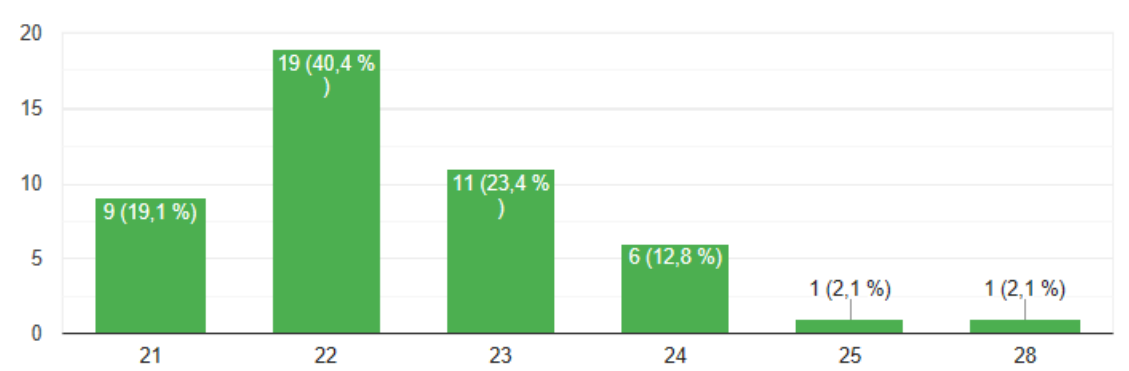

Figura 3. Distribución del alumnado en función de la edad

En cuanto a las preguntas donde el alumnado evaluaba la adquisición de competencias, se realizó un análisis estadístico de los datos obtenidos determinandose la distribución de 
frecuencias y las medidas de tendencia central para cada item (programa estadístico PSPP, versión 0.10.2), cuyos resultados se pueden ver en la Tabla 1.

Tabla 1. Valoración de la experiencia por el alumnado

\begin{tabular}{|c|c|c|c|c|c|c|}
\hline 1. Dificultad & 31 & 15 & 1 & 0 & 0 & $\mathbf{1 , 9 4}$ \\
\hline $\begin{array}{c}\text { 2. Adquisición } \\
\text { habilidades } \\
\text { laboratorio }\end{array}$ & 0 & 0 & 2 & 12 & 33 & $\mathbf{4 , 6 6}$ \\
$\begin{array}{c}\text { 3.Refuerzo/ } \\
\text { adquisición } \\
\text { conocimientos } \\
\text { urianálisis }\end{array}$ & 0 & 0 & 1 & 10 & 36 & $\mathbf{4 , 7 4}$ \\
\hline $\begin{array}{c}\text { 4. Utilidad urianálisis } \\
\text { en el ABP }\end{array}$ & 0 & 0 & 1 & 9 & 37 & $\mathbf{4 , 7 6}$ \\
\hline $\begin{array}{c}\text { 5. Adquisición } \\
\text { competencias } \\
\text { profesionalizantes }\end{array}$ & 1 & 1 & 2 & 12 & 31 & $\mathbf{4 , 5 1}$ \\
\hline \begin{tabular}{c} 
6. Satisfacción \\
\hline
\end{tabular} & 0 & 0 & 0 & 13 & 34 & $\mathbf{4 , 7 2}$ \\
\hline
\end{tabular}

De los resultados de las encuestas se desprende que el alumnado se mostró altamente satisfecho con la experiencia (valor medio $=4,72$ ) y no tuvo dificultad para realizarla (valor medio $=1,94)$. Las respuestas en cuanto a la evaluación de los objetivos planteados se centraron en dos aspectos fundamentales: a) la valoración de los estudiantes sobre la adquisición de destrezas/habilidades en el laboratorio y los conocimientos adquiridos sobre la realización del urianálisis que fue muy alta (valor medio=4,74). b) la valoración sobre la metodología del ABP para la adquisición de competencias profesionalizantes. La mayoría de los/as estudiantes consideró muy útil la integración de los resultados del urianálisis en el abordaje diagnóstico y resolución de los casos a los que se enfrentaron (valor medio $=4,76$ ). En general, consideró que los conocimientos adquiridos durante la experiencia, podían ser útiles o muy útiles en el desarrollo de su profesión en un futuro (valor medio=4,51).

Respecto a las observaciones llevadas a cabo por el profesorado durante la realización de la experiencia, el alumnado mostró mayor habilidad y autonomía en las destrezas laboratoriales conforme iban realizándose más urianálisis. Además, desarrollaron una confianza creciente en el abordaje de los casos clínicos durante el trabajo grupal en la consulta, apoyándose mucho más en el/la tutor/a en el abordaje diagnóstico de los primeros pacientes y siendo más autónomos en cuanto al análisis y la toma de decisiones en los últimos casos. Al final de la experiencia, la mayoría de los/as estudiantes fue capaz de 
identificar las alteraciones clínicas más relevantes y relacionarlas con un proceso patológico concreto, elaborar un diagnóstico diferencial y ordenarlo de forma razonada, establecer los pasos más adecuados para llegar al diagnostico definitivo del paciente y escoger las medidas terapéuticas a instaurar.

\section{Conclusiones}

En el diseño de esta experiencia se han integrado distintas metodologías que han permitido trabajar al mismo tiempo competencias específicas y transversales (Fernández, 2008). La sinergia entre el trabajo laboratorial, las sesiones clínicas con casos reales aplicando el método del ABP y el trabajo en pequeños grupos, ha permitido fomentar competencias genéricas: de tipo instrumental, al analizar e integrar los datos obtenidos en el laboratorio en la resolución de un caso clínico-problema; de tipo interpersonal, en cuanto al trabajo y la discusión de carácter grupal; y de tipo sistémico, al aplicar los conocimientos teóricos y prácticos adquiridos en un contexto real con pacientes reales (De La Cruz, 2007; Fernández, 2006). La evaluación realizada por los estudiantes demuestra que su percepción al realizar este tipo de experiencias es que, mediante las mismas, refuerzan y adquieren destrezas y habilidades específicas (profesionalizantes) que les van a ser muy útiles en su futuro profesional. El profesorado a su vez se mostró muy satisfecho con los resultados obtenidos, planteándose realizar esta experiencia en futuros cursos.

\section{Referencias}

BARROWS, H.S. and TAMBLYN, R.N. (1980). "Problem-based learning". New York: Springler Publishing Company.

CAROLYN, S and WEINSTEIN, N. (2012). "Practical Veterinary Urinalysis". First Edition. Chichester, UK: John Wiley and Sons editors.

DE LA CRUZ, Ma A. (2007). "Taller sobre el proceso de aprendizaje-enseñanza de competencias". Instituto de Ciencias de la Educación (ICE). Universidad de Zaragoza. Zaragoza: INO Reproducciones S.A.

ESCANERO, J.F. et al (2002). "Aprendizaje basado en la solución de problemas en fisiología circulatoria: el caso o problema como nexo de unión para la integración curricular" en Educación Médica, 5, (4), 191-4.

FERNANDEZ, A. (2006). "Metodologías activas para la formación de competencias" en Educatio siglo XXI, 24, 35-56.

FERNANDEZ, A. (2008). "Metodologías activas en la Universidad". Universidad de Zaragoza. En Diploma de Formación Pedagógica para el profesorado universitario. Instituto de Ciencias de la Educación (ICE).

WALTON, H.J. and MATTHEWS, M.B. (1989). "Essentials of problem-based learning" en Medical Education, 23, 542-58.

ANEXO I. Hoja-informe de urianálisis que el alumnado tuvo que completar durante las prácticas en el laboratorio. 


\section{HOJA DE URIANÁLISIS. HVZ. SERVICIO DE MEDICINA INTERNA- URINARIO}

Fecha:

Hora recogida:

Hora análisis:

$\mathbf{N}^{\text {o ficha: }}$

Paciente:

Edad:

Peso:

Patología posible: $\quad \square$ ITU $\square$ Urolitiasis $\square$ Insuficiencia renal

$\square$ PU/PD

Otra

Procedimiento obtención: $\square$ Cistocentesis $\quad \square$ Recogida directa $\square$ Sondaje

Aspecto físico de la orina y color:

DENSIDAD (refractometría):

Indice UPC:

TIRA REACTIVA:

$\square$ Orina Centrifugada

$\square$ Orina NO Centrifugada

\begin{tabular}{|l|l|l|l|l|l|}
\hline & - & $1+$ & $2+$ & $3+$ & $4+$ \\
\hline Sangre (eritrocitos) & & & & & \\
\hline Hemoglobina libre & & & & & \\
\hline Urobilinógeno & & & & & \\
\hline Bilirrubina & & & & & \\
\hline Proteínas & & & & & \\
\hline Nitritos & & & & & \\
\hline Cetonas & & & & & \\
\hline Glucosa & & & & & \\
\hline pH & & & & & \\
\hline Densidad & & & & & \\
\hline Leucocitos & & & & & \\
\hline
\end{tabular}

\section{SEDIMENTO:}

\begin{tabular}{|l|l|}
\hline Células hemáticas & \\
\hline Células epiteliales & \\
\hline Cilindros & \\
\hline Cristales & \\
\hline Bacterias & \\
\hline
\end{tabular}




\section{Otros}

ANEXO II. Encuesta de google que el alumnado tuvo que completar tras la realización de la práctica para evaluar distintos aspectos de la misma.

\section{ENCUESTA PARA LA VALORACIÓN DE ADQUISICIÓN DE COMPETENCIAS PROFESIONALES A TRAVÉS DEL URIANÁLISIS DENTRO DEL MÉTODO ABP.}

(Incorporación del urianálisis como técnica laboratorial en el abordaje diagnóstico de casos clinicos (ABP) para la adquisición de competencias profesionales)

Género:

Edad (años):

Curso académico actual:

\section{*Indica del 1 al 5 el grado de acuerdo o desacuerdo para cada uno de los enunciados,} siendo:

$1=$ Totalmente en desacuerdo

$2=$ En desacuerdo

$3=$ Neutral

$4=$ De acuerdo

$5=$ Totalmente de acuerdo

1. Esta práctica me ha resultado fácil de realizar *

2. La realización de esta práctica me ha ayudado a reforzar mis habilidades manuales en el laboratorio *

3. Esta práctica me ha ayudado a ampliar/reforzar mis conocimientos en cuánto a la técnica de urianálisis y a los nuevos equipamientos disponibles para su realización *

4. La realización e interpretación del urianálisis ha sido muy útil en la orientación/abordaje diagnóstico de los casos clínicos*

5. Los conocimientos adquiridos a lo largo de esta práctica pueden ser de gran ayuda para mi experiencia profesional en un futuro *

6. En general, la realización de esta práctica ha satisfecho mis expectativas * 